\title{
Log-Harnack Inequality for Stochastic Differential Equations in Hilbert Spaces and its Consequences*
}

\author{
Michael Röckner ${ }^{b), c)}$ and Fneg-Yu Wang ${ }^{a), d) \dagger}$ \\ ${ }^{a)}$ School of Math. Sci. \& Lab. Math. Com. Sys., Beijing Normal University, Beijing 100875, China \\ ${ }^{b)}$ Department of Mathematics, Bielefeld University, D-33501 Bielefeld, Germany \\ ${ }^{c)}$ Department of Mathematics and Statistics, Purdue University, W. Lafayette, IN 47907, USA \\ ${ }^{d)}$ Department of Mathematics, Swansea University, Singleton Park, SA2 8PP, UK
}

November 16, 2018

\begin{abstract}
A logarithmic type Harnack inequality is established for the semigroup of solutions to a stochastic differential equation in Hilbert spaces with non-additive noise. As applications, the strong Feller property as well as the entropy-cost inequality for the semigroup are derived with respect to the corresponding distance (cost function).
\end{abstract}

AMS subject Classification: 60J60, 58G32.

Keywords: Stochastic differential equation, log-Harnack inequality, strong Feller property, entropy-cost inequality.

\section{Introduction}

Under a curvature condition the second named author established the following type dimension-free Harnack inequality for diffusion semigroups on a Riemannian manifold $M$ (14]):

*Supported in part by WIMCS, NNSFC(10721091) and the 973-Project.

†Corresponding author. wangfy@bnu.edu.cn; F.Y.Wang@swansea.ac.uk 


$$
\left(P_{t} f\right)^{\alpha}(x) \leq\left(P_{t} f^{\alpha}\right)(x) \mathrm{e}^{c(t) \rho(x, y)^{2}}, \quad f \geq 0, t>0, \alpha>1, x, y \in M,
$$

where $c(t)>0$ is explicitly determined by $\alpha$ and the curvature lower bound. This inequality has been efficiently applied to the study of functional inequalities for the associated Dirichlet form, the hyper-/super-/ultracontractivity properties of the semigroup, strong Feller property as well as estimates on the heat kernel of the semigroup (cf. [8, 13, 15, 6] and references therein). To establish this inequality for diffusions with curvature unbounded below, a coupling method is developed in [1]. This method works also for infinite dimensional SPDE provided the noise is additive and non-degenrate, see e.g. [16, 9, 10, 11, 6] for Harnack inequalities for several different classes of SPDE. The aim of this paper is to extend the study to stochastic differential equations with non-additive noises.

Let us start from the following Itô stochastic differential equation on $\mathbb{R}^{n}$ :

$$
\mathrm{d} X_{t}=b\left(X_{t}\right) \mathrm{d} t+\sigma\left(X_{t}\right) \mathrm{d} B_{t},
$$

where $b: \mathbb{R}^{n} \rightarrow \mathbb{R}^{n}$ and $\sigma: \mathbb{R}^{n} \rightarrow \mathbb{R}^{n} \otimes \mathbb{R}^{n}$ are continuous, $B_{t}$ is the Brownian motion in $\mathbb{R}^{n}$. In this case the solution is a diffusion process with corresponding generator (Kolmogorov operator)

$$
L=\sum_{i, j=1}^{n} a_{i j}(x) \partial_{i} \partial_{j}+\sum_{i=1}^{n} b_{i}(x) \partial_{i},
$$

where $\left(a_{i j}\right)_{1 \leq i, j \leq n}=\frac{1}{2} \sigma^{*} \sigma$. If $a_{i j}$ and $b_{i}$ are regular enough such that Bakry-Emery's $\Gamma_{2}$ condition (see [2])

$$
\Gamma_{2}(f, f):=\frac{1}{2} L\langle a \nabla f, \nabla f\rangle-\langle a \nabla f, \nabla L f\rangle \geq-K\langle a \nabla f, \nabla f\rangle
$$

holds for all smooth $f$ and some constant $K$, then the curvature condition used in [14] holds for the Riemannian metric $\langle u, v\rangle_{a}:=\left\langle a^{-1} u, v\right\rangle, u, v \in \mathbb{R}^{n}$, induced by the diffusion coefficient. Thus, one derives the desired Harnack inequality for the associated diffusion semigroup. Theoretically one may use this argument to establish the Harnack inequality for non-constant $a$ also in infinite dimensions. For $n \rightarrow \infty$ condition (1.1) is, however, too complicated to verify or does not hold. This is the main reason why all existing results in this direction for infinite dimensional SDE are merely proved for additive noise (i.e. for the constant diffusion case).

In this paper we shall analyse the following log-Harnack inequality allowing the diffusion to be non-constant:

$$
P_{t} \log f(x) \leq \log P_{t} f(y)+\frac{K \rho_{a}(x, y)^{2}}{2\left(1-\mathrm{e}^{-2 K t}\right)}, \quad t>0, x, y \in \mathbb{R}^{n}, f>0,
$$


where $\rho_{a}$ is the distance induced by the metric $\langle\cdot, \cdot\rangle_{a}$. This inequality was first presented in the proof of [3, Lemma 4.2] under the $\Gamma_{2}$ condition (1.1) for $P_{t} f$ in place of $f$, which is crucial for the proof of the HWI inequality [3, Theorem 4.3]. We will see in Section 2 that this type of inequality can be derived by using a standard dissipative type condition which is explicit and dimension free. Combining this observation with an approximation argument, we are able to establish the inequality for infinite dimensional diffusions on Hilbert spaces, and furthermore derive the strong Feller property of the semigroup and entropy inequalities for the heat kernel.

We will work with the following semi-linear stochastic differential equation on a separable Hilbert space $(\mathbb{H},\langle\cdot, \cdot\rangle,\|\cdot\|)$ (cf. [7]):

$$
\mathrm{d} X_{t}=\left(A X_{t}+F\left(X_{t}\right)\right) \mathrm{d} t+\sigma\left(X_{t}\right) \mathrm{d} W_{t},
$$

where $W_{t}$ is a cylindrical Brownian motion on $\mathbb{H}$ on some filtered probability space $\left(\Omega, \mathcal{F}, \mathbb{P},\left(\mathcal{F}_{t}\right)\right) . \quad F$ is a Lipschtiz continuous function on $\mathbb{H}$, and $\sigma(x)=\tilde{\sigma}_{1}(x)+\tilde{\sigma}_{0}$ for a linear operator $\tilde{\sigma}_{0}$ and a Hilbert-Schmidt operator-valued function $\tilde{\sigma}_{1}$ such that $\sigma^{*} \sigma \geq \tilde{\sigma}_{0}^{2}$. We shall assume:

(H1) $A$ is a self-adjoint operator on $\mathbb{H}$ generating a contractive compact semigroup $T_{t}$. In this case $-A$ has discrete spectrum $0 \leq \lambda_{1} \leq \lambda_{2} \leq \cdots$ with corresponding eigenbasis $\left\{e_{i}\right\}_{i \geq 1}$ of $\mathbb{H}$. Let $\mathbb{H}_{n}=\operatorname{span}\left\{e_{1}, \cdots, e_{n}\right\}, n \geq 1$.

(H2) $\tilde{\sigma}_{0} e_{i}=q_{i} e_{i}$ for a sequence $\left\{q_{i}>0: i \geq 1\right\}$ such that $\sigma^{*} \sigma \geq \tilde{\sigma}_{0}^{2}$ and $\sum_{i=1}^{\infty} \frac{q_{i}^{2}}{1+\lambda_{i}}<\infty$.

(H3) $\tilde{\sigma}_{1}:=\sigma-\tilde{\sigma}_{0}$ is Hilbert-Schmidt and there exists a constant $C>0$ such that

$$
\|F(x)-F(y)\|+\left\|\tilde{\sigma}_{1}(x)-\tilde{\sigma}_{1}(y)\right\|_{H S} \leq C\|x-y\|, \quad x, y \in \mathbb{H} .
$$

(H4) There exists a constant $K \in \mathbb{R}$ such that

$$
2\left\langle F(x)-F(y), \tilde{\sigma}_{0}^{-2}(x-y)\right\rangle+\left\|\tilde{\sigma}_{0}^{-1}\left(\tilde{\sigma}_{1}(x)-\tilde{\sigma}_{1}(y)\right)\right\|_{H S}^{2} \leq K\left\|\tilde{\sigma}_{0}^{-1}(x-y)\right\|^{2}
$$

holds for all $x, y \in \mathbb{H}$ with $x-y \in \cup_{n=1}^{\infty} \mathbb{H}_{n}$.

We note that (H3) implies $(H 4)$ in case $\tilde{\sigma}_{0}$ and $\tilde{\sigma}_{0}^{-1}$ are both bounded. Obviously, (H1)-(H3) imply the existence and the uniqueness of the mild solution to (1.2), that is, for any $x \in \mathbb{H}$ there exists a unique $\mathbb{H}$ valued adapted process $X_{t}$, which is continuous in $L^{2}(\Omega, \mathbb{P})$, such that (cf. [7])

$$
X_{t}=T_{t} x+\int_{0}^{t} T_{t-s} F\left(X_{s}\right) \mathrm{d} s+\int_{0}^{t} T_{t-s} \sigma\left(X_{s}\right) \mathrm{d} W_{s} .
$$

Let $P_{t}$ be the associated Markov semigroup, i.e. 


$$
P_{t} f(x)=\mathbb{E} f\left(X_{t}\right), \quad f \in \mathscr{B}_{b}(\mathbb{H}),
$$

where $\mathscr{B}_{b}(\mathbb{H})$ is the set of all bounded measurable functions on $\mathbb{H}$. In this paper we shall establish a log-Harnack inequality for $P_{t}$ by using $(H 4)$ in place of the $\Gamma_{2}$ condition.

Theorem 1.1. If $(H 1)-(H 4)$ hold then for any strictly positive $f \in \mathscr{B}_{b}(\mathbb{H})$,

$$
P_{t} \log f(x) \leq \log P_{t} f(y)+\frac{K\left\|\tilde{\sigma}_{0}^{-1}(x-y)\right\|^{2}}{2\left(1-\mathrm{e}^{-K t}\right)}, \quad t>0, x, y \in \mathbb{H},
$$

where $\left\|\tilde{\sigma}_{0}^{-1} x\right\|^{2}:=\sum_{i=1}^{\infty} q_{i}^{-2}\left\langle x, e_{i}\right\rangle^{2} \in[0, \infty]$.

As applications of Theorem 1.1, we have the following results on the strong Feller property, heat kernel inequality and entropy-cost inequality. To state these results, let us introduce some notions. Let

$$
\mathbb{H}_{0}=\left\{x \in \mathbb{H}:\|x\|_{0}:=\left\|\tilde{\sigma}_{0}^{-1} x\right\|<\infty\right\} .
$$

We call $P_{t} \mathbb{H}_{0}$-strong Feller if for any $f \in \mathscr{B}_{b}(\mathbb{H})$,

$$
\lim _{\|y-x\|_{0} \rightarrow 0} P_{t} f(y)=P_{t} f(x), \quad x \in \mathbb{H} .
$$

When $\tilde{\sigma}_{0}^{-1}$ is bounded then $\mathbb{H}_{0}=\mathbb{H}$ and $\mathbb{H}_{0}$-strong Feller, implies $\mathbb{H}$-strong Feller. Next, let $P_{t}$ be $\mathbb{H}_{0}$-strong Feller and let $\mu$ be a probability measure on $\mathbb{H}$ such that for some $C, \alpha>0$,

$$
\int P_{t} f \mathrm{~d} \mu \leq C e^{\alpha t} \int f \mathrm{~d} \mu \text { for all } f \in \mathscr{B}_{b}(\mathbb{H}), f \geq 0,
$$

(which holds e.g. if $\mu$ is $P_{t}$-invariant). Such measures always exist. Take e.g. for $x_{0} \in \mathbb{H}$, $\mu(d y):=\int_{0}^{\infty} e^{-s} P_{s}\left(x_{0}, d y\right) d s$. Then $\mu$ satisfies (1.4) with $\alpha=1=C$. Suppose that $\mu$ is fully supported on $\mathbb{H}_{0}$, i.e. $\mu(U)>0$ for every nonempty $\|\cdot\|_{0}$-open set $U \subset \mathbb{H}_{0}$. Then it is easy to see that for every $x \in \mathbb{H}_{0}, P_{t}(x, d y)$ has a transition density $p_{t}(x, y)$ with respect to $\mu$.

Remark. Obviously $\left(\mathbb{H}_{0},\|\cdot\|_{0}\right)$ is separable. Hence there exists $\mu$ as in (1.4) fully supported on $\mathbb{H}_{0}$. Indeed, take a countable $\|\cdot\|_{0}$-dense subset $\left\{x_{n} \mid n \in \mathbb{N}\right\}$ of $\mathbb{H}_{0}$. Then

$$
\mu(d y):=\sum_{n=1}^{\infty} \frac{1}{2^{n}} \int_{0}^{\infty} e^{-s} P_{s}\left(x_{n}, d y\right) \mathrm{d} s
$$

is a probability measure on $\mathbb{H}$, satisfying (1.4). Furthermore, if $U \subset \mathbb{H}_{0}$ is $\|\cdot\|_{0}$-open such that $\mu(U)=0$. Then for $\varphi(x):=\inf \left\{\|x-y\|_{0}: y \in U^{c}\right\}$ we have

$$
\int \varphi \mathrm{d} \mu=0
$$


Hence by a diagonal argument we can find a zero sequence $\left(t_{k}\right)_{k \in \mathbb{N}}$ such that $P_{t_{k}} \varphi\left(x_{n}\right)=0$ for all $k, n \in \mathbb{N}$. Taking $k \rightarrow \infty$ we obtain $\varphi\left(x_{n}\right)=0$ for all $n \in \mathbb{N}$. But if $U \neq \emptyset$, then $x_{n_{0}} \in U$ for some $n_{0} \in \mathbb{N}$, so $\varphi\left(x_{n_{0}}\right)>0$. This contradiction shows that $U=\emptyset$.

Finally, for two probability measures $\mu_{1}, \mu_{2}$ on $\mathbb{H}$, let $W_{0}\left(\mu_{1}, \mu_{2}\right)$ be the $L^{2}$-Wasserstein distance or $L^{2}$-transportation cost between them with respect to the cost function $(x, y) \mapsto$ $\|x-y\|_{0}$. More precisely, with $\mathscr{C}\left(\mu_{1}, \mu_{2}\right)$ denoting the set of all couplings of $\mu_{1}$ and $\mu_{2}$, we have

$$
W_{0}\left(\mu_{1}, \mu_{2}\right)^{2}=\inf _{\pi \in \mathscr{C}\left(\mu_{1}, \mu_{2}\right)} \int_{\mathbb{H} \times \mathbb{H}} \|\left(\tilde{\sigma}_{0}^{-1}(x-y) \|^{2} \pi(\mathrm{d} x, \mathrm{~d} y) .\right.
$$

Corollary 1.2. Let (H1)- (H4) hold. Then:

(1) For any $t>0, P_{t}$ is $\mathbb{H}_{0}$-strong Feller. Let $\mu$ be $P_{t}$-subinvariant (i.e., (1.4) holds with $C=1, \alpha=0$ ). Then (1.3) holds for all $\mu$-exponentially integrable functions $f$.

(2) Let $\mu$ be as in (1.4) above, fully supported on $\mathbb{H}_{0}$. Then for every $x \in \mathbb{H}_{0}, P_{t}(x, d y)$ has a transition density $p_{t}(x, y)$ satisfying the following entropy inequality

$$
\int_{\mathbb{H}} p_{t}(x, z) \log p_{t}(x, z) \mu(\mathrm{d} z) \leq \log C+\alpha t-\log \int_{\mathbb{H}} \exp \left[-\frac{K\|x-y\|_{0}^{2}}{2\left(1-\mathrm{e}^{-K t}\right)}\right] \mu(\mathrm{d} y), \quad t>0, x \in \mathbb{H} .
$$

(3) Let $\mu$ be $P_{t}$-subinvariant. Then the following entropy-cost inequality holds for the adjoint operator $P_{t}^{*}$ of $P_{t}$ in $L^{2}(\mu)$ :

$$
\mu\left(\left(P_{t}^{*} f\right) \log P_{t}^{*} f\right) \leq \frac{K}{2\left(1-\mathrm{e}^{-K t}\right)} W_{0}(f \mu, \mu)^{2}, \quad t>0, f \geq 0, \mu(f)=1 .
$$

In Section 2 we shall prove the log-Harnack inequality for diffusion semigroups on $\mathbb{R}^{n}$ and then we extend this to an infinite dimensional setting in Section 3 by finite-dimensional approximations. Finally, Corollary 1.2 will be proved in Section 4.

\section{Log-Harnack inequality on $\mathbb{R}^{n}$}

Consider the following SDE on $\mathbb{R}^{n}$ :

$$
\mathrm{d} X_{t}=b\left(X_{t}\right) \mathrm{d} t+\sigma\left(X_{t}\right) \mathrm{d} B_{t},
$$

where $B_{t}$ is Brownian motion on $\mathbb{R}^{n}, b: \mathbb{R}^{n} \rightarrow \mathbb{R}^{n}$ and $\sigma: \mathbb{R}^{n} \rightarrow \mathbb{R}^{n} \otimes \mathbb{R}^{n}$ are locally Lipschitzian and of at most linear growth. Hence the equation has a unique strong solution, which is non-explosive. Let $\tilde{\sigma}_{0}$ be a (strictly) positive definite symmetric matrix such that $\sigma^{*} \sigma \geq \tilde{\sigma}_{0}^{2}$. Assume that 


$$
\|\left(\tilde { \sigma } _ { 0 } ^ { - 1 } \left(\sigma(x)-\sigma(y)\left\|_{H S}^{2}+2\left\langle\tilde{\sigma}_{0}^{-1}(b(x)-b(y)), \tilde{\sigma}_{0}^{-1}(x-y)\right\rangle \leq K\right\| \tilde{\sigma}_{0}^{-1}(x-y) \|^{2}, \quad x, y \in \mathbb{R}^{n}\right.\right.
$$

holds for some constant $K \in \mathbb{R}$.

Theorem 2.1. Assume (2.2) and that the solution to (2.1) is non-explosive. Then the associated Markov semigroup $P_{t}$ satisfies

$$
P_{t} \log f(x) \leq \log P_{t} f(y)+\frac{K\left\|\tilde{\sigma}_{0}^{-1}(x-y)\right\|^{2}}{2\left(1-\mathrm{e}^{-K t}\right)}, \quad t>0, x, y \in \mathbb{R}^{n}
$$

for all $f \in \mathscr{B}_{b}\left(\mathbb{R}^{n}\right), f \geq 0$.

By a standard approximation argument we can assume that $f \in C_{b}^{\infty}\left(\mathbb{R}^{n}\right)$. Furthermore, we can approximate $b, \sigma$ by smooth $b_{n}, \sigma_{n}$ such that the corresponding semigroups converge pointwise on $f \in C_{b}^{\infty}\left(\mathbb{R}^{n}\right)$ and such that $P_{t} C_{b}^{2} \subset C_{b}^{2}$ for all $t>0$.

To prove the log-Harnack inequality, we need the following gradient estimate on $P_{t}$.

Lemma 2.2. Under the assumptions of Theorem 2.1 we have

$$
\left\|\tilde{\sigma}_{0} \nabla P_{t} f\right\|^{2}(x) \leq \mathrm{e}^{K t} P_{t}\left\|\tilde{\sigma}_{0} \nabla f\right\|^{2}(x), \quad f \in C_{b}^{1}\left(\mathbb{R}^{n}\right), x \in \mathbb{R}^{n} .
$$

Proof. For $x, y \in \mathbb{R}^{n}$, let $X_{t}$ and $Y_{t}$ be the solutions to (2.1) with $X_{0}=x$ and $Y_{0}=y$ respectively. By Itô's formula and (2.2) we obtain

$$
\begin{aligned}
& \mathrm{d}\left\|\tilde{\sigma}_{0}^{-1}\left(X_{t}-Y_{t}\right)\right\|^{2}=2\left\langle\tilde{\sigma}_{0}^{-1}\left(X_{t}-Y_{t}\right), \tilde{\sigma}_{0}^{-1}\left(\sigma\left(X_{t}\right)-\sigma\left(Y_{t}\right)\right) \mathrm{d} B_{t}\right\rangle \\
& +\left\{\|\left(\tilde{\sigma}_{0}^{-1}\left(\sigma\left(X_{t}\right)-\sigma\left(Y_{t}\right)\right) \|_{H S}^{2}+2\left\langle\tilde{\sigma}_{0}^{-1}\left(b\left(X_{t}\right)-b\left(Y_{t}\right)\right), \tilde{\sigma}_{0}^{-1}\left(X_{t}-Y_{t}\right)\right\rangle\right\} \mathrm{d} t\right. \\
& \leq 2\left\langle\tilde{\sigma}_{0}^{-1}\left(X_{t}-Y_{t}\right), \tilde{\sigma}_{0}^{-1}\left(\sigma\left(X_{t}\right)-\sigma\left(Y_{t}\right)\right) \mathrm{d} B_{t}\right\rangle+K\left\|\tilde{\sigma}_{0}^{-1}\left(X_{t}-Y_{t}\right)\right\|^{2} \mathrm{~d} t .
\end{aligned}
$$

Since the solution to (2.1) is non-explosive, this implies

$$
\mathbb{E}\left\|\tilde{\sigma}_{0}^{-1}\left(X_{t}-Y_{t}\right)\right\|^{2} \leq \mathrm{e}^{K t}\left\|\tilde{\sigma}_{0}^{-1}(x-y)\right\|^{2} .
$$

Therefore,

$$
\begin{aligned}
\left\|\tilde{\sigma}_{0} \nabla P_{t} f\right\|^{2}(x) & =\limsup _{y \rightarrow x} \frac{\left|P_{t} f(y)-P_{t} f(x)\right|^{2}}{\left\|\tilde{\sigma}_{0}^{-1}(x-y)\right\|^{2}}=\limsup _{y \rightarrow x}\left(\frac{\mathbb{E}\left(f\left(Y_{t}\right)-f\left(X_{t}\right)\right)}{\left\|\tilde{\sigma}_{0}^{-1}(x-y)\right\|}\right)^{2} \\
& \leq \limsup _{y \rightarrow x}\left(\mathbb{E} \frac{\left|f\left(Y_{t}\right)-f\left(X_{t}\right)\right|^{2}}{\left\|\tilde{\sigma}_{0}^{-1}\left(Y_{t}-X_{t}\right)\right\|^{2}}\right) \frac{\mathbb{E}\left\|\tilde{\sigma}_{0}^{-1}\left(X_{t}-Y_{t}\right)\right\|^{2}}{\left\|\tilde{\sigma}_{0}^{-1}(x-y)\right\|^{2}} \\
& \leq \mathrm{e}^{K t} \mathbb{E}\left\|\tilde{\sigma}_{0} \nabla f\right\|^{2}\left(X_{t}\right) .
\end{aligned}
$$

This implies the desired gradient estimate. 
Proof of Theorem [2.1. We may assume $f \geq 1$. For fixed $x \in \mathbb{R}^{n}$, let $X_{0}=x$. By Itô's formula we have

$$
\begin{aligned}
\mathrm{d} \log P_{t-s} f\left(X_{s}\right) & =\left\langle\nabla \log P_{t-s} f\left(X_{s}\right), \sigma\left(X_{s}\right) \mathrm{d} B_{s}\right\rangle+L \log P_{t-s} f\left(X_{s}\right) \mathrm{d} s-\frac{L P_{t-s} f}{P_{t-s} f}\left(X_{s}\right) \mathrm{d} s \\
& =\left\langle\nabla \log P_{t-s} f\left(X_{s}\right), \sigma\left(X_{s}\right) \mathrm{d} B_{s}\right\rangle-\frac{1}{2}\left\|\sigma \nabla \log P_{t-s} f\right\|^{2}\left(X_{s}\right) \mathrm{d} s .
\end{aligned}
$$

Letting

$$
\tau_{k}=\inf \left\{t \geq 0:\left\|X_{t}\right\| \geq k\right\}, \quad k \geq 1
$$

we obtain

$$
\mathbb{E} \log P_{t-s \wedge \tau_{k}} f\left(X_{s \wedge \tau_{k}}\right)-P_{t} f(x)=-\frac{1}{2} \mathbb{E} \int_{0}^{s \wedge \tau_{k}}\left\|\sigma \nabla \log P_{t-r} f\right\|^{2}\left(X_{r}\right) \mathrm{d} r .
$$

Since the process is non-explosive, we have $\tau_{k} \rightarrow \infty$. Thus, due to the dominated convergence theorem, as $k \rightarrow \infty$ the left-hand side goes to $P_{s} \log P_{t-s} f(x)-\log P_{t} f(x)$, while by the monotone convergence theorem, the right-hand side goes to $-\frac{1}{2} \int_{0}^{s} P_{r}\left\|\sigma \nabla \log P_{t-r} f\right\|^{2}(x) \mathrm{d} r$. So, $\int_{0}^{t} P_{r}\left\|\sigma \nabla \log P_{t-r} f\right\|^{2}(x) \mathrm{d} r<\infty$ and

$$
P_{s} \log P_{t-s} f(x)-\log P_{t} f(x)=-\frac{1}{2} \int_{0}^{s} P_{r}\left\|\sigma \nabla \log P_{t-r} f\right\|^{2}(x) \mathrm{d} r, \quad s \in[0, t] .
$$

Now, for fixed $x, y \in \mathbb{R}^{n}, t>0$, let

$$
x_{s}=(x-y) h_{s}+y, \quad s \in[0, t],
$$

where $h \in C^{1}([0, t], \mathbb{R})$ such that $h_{0}=0$ and $h_{t}=1$. By Lemma 2.2. (2.3) and noting that $\sigma^{*} \sigma \geq \tilde{\sigma}_{0}^{2}$, we have, since $s \mapsto P_{s} \log P_{t-s} f\left(x_{s}\right)$ is absolutely continuous by Lemma 2.2, that

$$
\begin{aligned}
& P_{t} \log f(x)-\log P_{t} f(y) \\
= & \int_{0}^{t} \frac{\mathrm{d}}{\mathrm{d} s}\left(P_{s} \log P_{t-s} f\right)\left(x_{s}\right) \mathrm{d} s \\
= & -\frac{1}{2} \int_{0}^{t}\left\{P_{s}\left\|\sigma \nabla \log P_{t-s} f\right\|^{2}\left(x_{s}\right)+h_{s}^{\prime}\left\langle x-y, \nabla P_{s} \log P_{t-s} f\right\rangle\left(x_{s}\right)\right\} \mathrm{d} s \\
\leq & -\frac{1}{2} \int_{0}^{t}\left\{\mathrm{e}^{-K s}\left\|\tilde{\sigma}_{0} \nabla P_{s} \log P_{t-s} f\right\|^{2}\left(x_{s}\right)+\left|h_{s}^{\prime}\right| \cdot\left\|\tilde{\sigma}_{0}^{-1}(x-y)\right\| \cdot\left\|\tilde{\sigma}_{0} \nabla P_{s} \log P_{t-s} f\right\|\right\} \mathrm{d} s \\
\leq & \frac{\left\|\tilde{\sigma}_{0}^{-1}(x-y)\right\|^{2}}{2} \int_{0}^{t} \mathrm{e}^{K s}\left|h_{s}^{\prime}\right|^{2} \mathrm{~d} s .
\end{aligned}
$$


Letting

$$
h_{s}=\frac{1-\mathrm{e}^{-K s}}{1-\mathrm{e}^{-K t}}, \quad s \in[0, t]
$$

we complete the proof.

\section{Proof of Theorem 1.1}

For any $n \geq 1$, let $\pi_{n}: \mathbb{H} \rightarrow \mathbb{H}_{n}:=\operatorname{span}\left\{e_{1}, \cdots, e_{n}\right\}$ be the orthogonal projection. Let $W_{t}^{n}=\pi_{n} W_{t}, A_{n}=\pi_{n} A, \sigma_{n}=\pi_{n} \sigma, \sigma_{i, n}=\pi_{n} \tilde{\sigma}_{i}(i=0,1)$, and $F_{n}=\pi_{n} F$. By $(H 1)$ and (H2) we have

$$
A_{n} x=A x, \quad \sigma_{0, n} x=\tilde{\sigma}_{0} x, \quad x \in \mathbb{H}_{n} .
$$

Consider the following SDE on $\mathbb{H}_{n}$ :

$$
\mathrm{d} X_{t}^{n}=\left(A_{n} X_{t}^{n}+F_{n}\left(X_{t}^{n}\right)\right) \mathrm{d} t+\sigma_{n}\left(X_{t}^{n}\right) \mathrm{d} W_{t}^{n}, \quad X_{0}^{n}=\pi_{n} X_{0} .
$$

By $(H 3)$ we see that both $b_{n}(x):=A_{n} x+F_{n}(x)$ and $\sigma_{n}(x)$ are Lipschitzian in $x \in \mathbb{H}_{n}$. So, this equation has a unique solution. Let $P_{t}^{n}$ be the associated Markov semigroup. Moreover, by $(H 4)$, since $A \leq 0$ and by (3.1) we have

$$
\begin{aligned}
& 2\left\langle\sigma_{0, n}^{-1}\left(b_{n}(x)-b_{n}(y)\right), \sigma_{0, n}^{-1}(x-y)\right\rangle+\left\|\sigma_{0, n}^{-1}\left(\sigma_{n}(x)-\sigma_{n}(y)\right)\right\|_{H S}^{2} \\
& \leq 2\left\langle\tilde{\sigma}_{0}^{-1}(F(x)-F(y)), \tilde{\sigma}_{0}^{-1}(x-y)\right\rangle+\left\|\tilde{\sigma}_{0}^{-1}(\sigma(x)-\sigma(y))\right\|_{H S}^{2} \\
& \leq K\left\|\tilde{\sigma}_{0}^{-1}(x-y)\right\|^{2}, \quad x, y \in \mathbb{H}_{n} .
\end{aligned}
$$

Thus, Theorem 2.1 implies that for $f \in C_{b}\left(\mathbb{H}_{n}\right)$

$$
P_{t}^{n} \log f(x) \leq \log P_{t}^{n} f(y)+\frac{K\left\|\tilde{\sigma}_{0}^{-1}(x-y)\right\|^{2}}{2\left(1-\mathrm{e}^{-K t}\right)}, \quad t>0, x, y \in \mathbb{H}^{n} .
$$

So, to derive the inequality for $P_{t}$, we need only to prove that

$$
\lim _{n \rightarrow \infty} \mathbb{E}\left\|X_{t}^{n}-X_{t}\right\|^{2}=0, \quad X_{0}=x \in \bigcup_{n \geq 1} \mathbb{H}_{n}
$$

Indeed, this implies that for any Lipschitzian function $f$ on $\mathbb{H}$, such that $f=f \circ \pi_{m}$ for some $m \in \mathbb{N}$ 


$$
\lim _{n \rightarrow \infty}\left|P_{t} f(x)-P_{t}^{n} f\left(\pi_{n} x\right)\right| \leq\|f\|_{L i p} \lim _{n \rightarrow \infty} \mathbb{E}\left\|X_{t}-X_{t}^{n}\right\|=0, \quad \forall x \in \bigcup_{n \geq 1} \mathbb{H}_{n}
$$

Therefore, by letting $n \rightarrow \infty$ in (3.2) we derive the desired log-Harnack inequality for such Lipschitzian functions first for $x \in \bigcup_{n \geq 1} \mathbb{H}_{n}$, but then since this set is dense in $\mathbb{H}$ and $P_{t} f$ is continuous for all such $f$, hence also for $\log (f+\varepsilon)$, we obtain it for all $x \in \mathbb{H}$. Finally, we extend it for all $f \in \mathscr{B}_{b}(\mathbb{H})$ by the monotone class theorem.

In order to prove (3.3), let

$$
Y_{t}=\int_{0}^{t} T_{t-s} \tilde{\sigma}_{0} \mathrm{~d} W_{s}, \quad Y_{t}^{n}=\pi_{n} Y_{t}=\int_{0}^{t} \mathrm{e}^{(t-s) A_{n}} \sigma_{0, n} \mathrm{~d} W_{s}^{n}, \quad t>0 .
$$

By $(H 2)$ we have

$$
\sup _{s \in[0, t]} \mathbb{E}\left\|Y_{s}\right\|^{2}<\infty
$$

so that the dominated convergence theorem implies

$$
\lim _{n \rightarrow \infty} \mathbb{E}\left\|Y_{t}-Y_{t}^{n}\right\|^{2}=0, \quad \lim _{n \rightarrow \infty} \int_{0}^{t} \mathbb{E}\left\|Y_{s}-Y_{s}^{n}\right\|^{2} \mathrm{~d} s=0
$$

Let

$$
Z_{t}=X_{t}-Y_{t}, \quad Z_{t}^{n}=X_{t}^{n}-Y_{t}^{n}
$$

By (3.4) it suffices to prove

$$
\lim _{n \rightarrow \infty} \mathbb{E}\left\|Z_{t}-Z_{t}^{n}\right\|^{2}=0
$$

We have

$$
\begin{aligned}
& \mathrm{d} Z_{t}=\left(A Z_{t}+F\left(Z_{t}+Y_{t}\right)\right) \mathrm{d} t+\sigma_{1}\left(Z_{t}+Y_{t}\right) \mathrm{d} W_{t}, \\
& \mathrm{~d} Z_{t}^{n}=\left(A_{n} Z_{t}^{n}+F_{n}\left(Z_{t}^{n}+Y_{t}^{n}\right)\right) \mathrm{d} t+\sigma_{1, n}\left(Z_{t}^{n}+Y_{t}^{n}\right) \mathrm{d} W_{t}^{n} .
\end{aligned}
$$

To be precise, (3.6) is first meant in the mild sense. But by our assumptions it also has a unique variational solution (see e.g. [12]). Since both are analytically weak solutions and these are unique (see e.g. the recent paper [4, where uniquenss of analytically weak solutions is proved for an even more general class of equations), we see that $Z_{t}$ defined above solves (3.6) in the variational sense, so that Itô's formula applies to $\left\|Z_{t}-Z_{t}^{n}\right\|^{2}$. Due to (3.1) we have

$$
\begin{aligned}
\mathrm{d}\left(Z_{t}-Z_{t}^{n}\right)= & \left(A\left(Z_{t}-Z_{t}^{n}\right)+F\left(X_{t}\right)-F_{n}\left(X_{t}^{n}\right)\right) \mathrm{d} t \\
& +\left(\sigma_{1}\left(X_{t}\right)-\pi_{n} \sigma_{1}\left(X_{t}^{n}\right)\right) \mathrm{d} W_{t}^{n}+\sigma_{1}\left(X_{t}\right) \mathrm{d}\left(W_{t}-W_{t}^{n}\right) .
\end{aligned}
$$


So, by Iô's formula and $(H 3)$ we obtain

$$
\begin{aligned}
& \mathrm{d}\left\|Z_{t}-Z_{t}^{n}\right\|^{2} \leq C_{1}\left(\left\|F\left(X_{t}\right)-F_{n}\left(X_{t}^{n}\right)\right\| \cdot\left\|X_{t}-X_{t}^{n}\right\|\right. \\
&\left.+\left\|\sigma_{1}\left(X_{t}\right)-\pi_{n} \sigma_{1}\left(X_{t}^{n}\right)\right\|_{H S}^{2}+\sum_{i>n}\left\|\sigma_{1}\left(X_{t}\right) e_{i}\right\|^{2}\right) \mathrm{d} t \\
& \leq C_{2}\left(\left\|Z_{t}-Z_{t}^{n}\right\|^{2}+\left\|Y_{t}-Y_{t}^{n}\right\|^{2}+\left\|\left(1-\pi_{n}\right) F\left(X_{t}\right)\right\|^{2}+\sum_{i>n}\left\|\sigma_{1}\left(X_{t}\right) e_{i}\right\|^{2}\right) \mathrm{d} t
\end{aligned}
$$

for some constants $C_{1}, C_{2}>0$. Since by $(H 3)$ we have

$$
\sup _{s \in[0, t]} \mathbb{E}\left(\left\|F\left(X_{s}\right)\right\|^{2}+\left\|\sigma_{1}\left(X_{s}\right)\right\|_{H S}^{2}\right) \leq C_{3} \sup _{s \in[0, t]}\left(1+\mathbb{E}\left\|X_{s}\right\|^{2}\right)<\infty,
$$

by the dominated convergence theorem

$$
\varepsilon_{n}:=C_{2} \mathbb{E} \int_{0}^{t}\left(\left\|Y_{s}-Y_{s}^{n}\right\|^{2}+\left\|\left(1-\pi_{n}\right) F\left(X_{s}\right)\right\|^{2}+\sum_{i>n}\left\|\sigma_{1}\left(X_{s}\right) e_{i}\right\|^{2}\right) \mathrm{d} s \rightarrow 0
$$

as $n \rightarrow \infty$. Thus, it follows from (3.8) that

$$
\lim _{n \rightarrow \infty} \mathbb{E} \mathrm{e}^{-C_{2} t}\left\|Z_{t}-Z_{t}^{n}\right\|^{2} \leq \lim _{n \rightarrow \infty} \varepsilon_{n}=0 .
$$

Therefore, (3.5) holds.

\section{Proof of Corollary 1.2}

It is sufficient to prove (1.3) for nonnegative $f \in \mathscr{B}_{b}(\mathbb{H})$. Applying the log-Harnack inequality in Theorem 1.1 for $1+\varepsilon f$ in place of $f$, we obtain from the elementary inequality $r \leq \log (1+r)+r^{2}, r \geq 0$,

$P_{t} f(y)-\varepsilon\|f\|_{\infty}^{2} \leq P_{t} \frac{\log (1+\varepsilon f)}{\varepsilon}(y) \leq \frac{1}{\varepsilon} \log \left(1+\varepsilon P_{t} f(x)\right)+\frac{c_{t}\|x-y\|_{0}^{2}}{\varepsilon}, \quad \varepsilon>0, x, y \in \mathbb{H}$,

where $c_{t}:=\frac{K}{2\left(1-\mathrm{e}^{-K t}\right)}$. Letting first $y \rightarrow x$ in $\|\cdot\|_{0}$ and then $\varepsilon \rightarrow 0$, we obtain

$$
\limsup _{\|y-x\|_{0} \rightarrow 0} P_{t} f(y) \leq P_{t} f(x) .
$$

Similarly, we have

$$
P_{t} \frac{\log (1+\varepsilon f)}{\varepsilon}(x)-\frac{c_{t}\|x-y\|_{0}^{2}}{\varepsilon} \leq \frac{1}{\varepsilon} \log \left(1+\varepsilon P_{t} f(y)\right) \leq P_{t} f(y) .
$$


Letting first $y \rightarrow x$ in $\|\cdot\|_{0}$ then $\varepsilon \rightarrow 0$, we arrive at

$$
P_{t} f(x) \leq \liminf _{\|x-y\|_{0} \rightarrow 0} P_{t} f(y)
$$

Therefore, $P_{t} f$ is $\|\cdot\|_{0}$ continuous. The second part of assertion (1.1) is then an easy consequence.

Now, let $p_{t}(x, y)$ be the transition density of $P_{t}$ with respect to $\mu$. By Theorem 1.1, for any positive $f \in \mathscr{B}_{b}(\mathbb{H})$ we have

$$
\mathrm{e}^{P_{t} \log f(x)} \leq \exp \left[\frac{K\|x-y\|_{0}^{2}}{2\left(1-\mathrm{e}^{-K t}\right)}\right] P_{t} f(y), \quad x, y \in \mathbb{H} .
$$

Thus,

$$
\mathrm{e}^{P_{t} \log f(x)} \int_{\mathbb{H}} \exp \left[-\frac{K\|x-y\|_{0}^{2}}{2\left(1-\mathrm{e}^{-K t}\right)}\right] \mu(\mathrm{d} y) \leq \int_{\mathbb{H}} P_{t} f(y) \mu(\mathrm{d} y)=C \mathrm{e}^{\alpha t} \mu(f) .
$$

For fixed $x \in \mathbb{H}$, applying this inequality to $f=n \wedge p_{t}(x, \cdot)$ then letting $n \rightarrow \infty$, we obtain

$$
\mathrm{e}^{\int_{\mathbb{H}} p_{t}(x, z) \log p_{t}(x, z) \mu(\mathrm{d} z)} \int_{\mathbb{H}} \exp \left[-\frac{K\|x-y\|_{0}^{2}}{2\left(1-\mathrm{e}^{-K t}\right)}\right] \mu(\mathrm{d} y) \leq C \mathrm{e}^{\alpha t} .
$$

This implies (2).

By approximations it remains to prove (3) for bounded positive $f$ with $\mu(f)=1$. By Theorem 1.1 for $P_{t}^{*} f$ in place of $f$, we obtain

$$
P_{t} \log P_{t}^{*} f(x) \leq \log P_{t} P_{t}^{*} f(y)+\frac{K\|x-y\|_{0}^{2}}{2\left(1-\mathrm{e}^{-K t}\right)}, \quad x, y \in \mathbb{H} .
$$

So, for any $\pi \in \mathscr{C}(f \mu, \mu)$, integrating both sides with respect to $\pi(\mathrm{d} x, \mathrm{~d} y)$ we arrive at

$$
\mu\left(\left(P_{t}^{*} f\right) \log P_{t}^{*}\right) \leq \mu\left(\log P_{t} P_{t}^{*} f\right)+\frac{K}{2\left(1-\mathrm{e}^{-K t}\right)} \int_{\mathbb{H} \times \mathbb{H}}\|x-y\|_{0}^{2} \pi(\mathrm{d} x, \mathrm{~d} y) .
$$

Since Jensen's inequality implies

$$
\mu\left(\log P_{t} P_{t}^{*} f\right) \leq \log \mu\left(P_{t} P_{t}^{*} f\right)=\log \mu(f)=0,
$$

this implies the desired entropy-cost inequality.

\section{References}

[1] M. Arnaudon, A. Thalmaier and F.-Y. Wang, Harnack inequality and heat kernel estimates on manifolds with curvature unbounded below, Bull. Sci. Math. 130, no. 3, 223-233, 2006. 
[2] D. Bakry and M. Emery, Hypercontractivité de semi-groupes de diffusion, C. R. Acad. Sci. Paris. Sér. I Math. 299(1984), 775-778.

[3] S. G. Bobkov, I. Gentil and M. Ledoux, Hypercontractivity of Hamilton-Jacobi equations, J. Math. Pures Appl. 80(2001), 669-696.

[4] Barbu, Marinelli, Röckner, Preprint

[5] G. Da Prato and M. Röckner, Singular dissipative stochastic equations in Hilbert spaces, Probab. Theory Relat. Fields, 124, 2, 261-303, 2002.

[6] G. Da Prato, M. Röckner and F.-Y. Wang, Singular stochastic equations on Hilbert spaces: Harnack inequalities for their transition semigroups, J. Funct. Anal. 257 (2009), no. 4, 992-1017.

[7] G. Da Prato and J. Zabczyk, Stochastic equations in infinite dimensions, Cambridge University Press, 1992.

[8] F.-Z. Gong and F.-Y. Wang, Heat kernel estimates with application to compactness of manifolds, Q. J. Math. 52 (2001), no. 2, 171-180.

[9] W. Liu, Doctor-Thesis, Bielefeld University, 2009.

[10] W. Liu and F.-Y. Wang, Harnack inequality and strong Feller property for stochastic fast diffusion equations, J. Math. Anal. Appl. 342(2008), 651-662.

[11] S.-X. Ouyang, Doctor-Thesis, Bielefeld University, 2009.

[12] C. Prevot and M. Röckner, A concise course on stochastic partial differential equations, Lecture Notes in Mathematics, Springer, 2007.

[13] M. Röckner and F.-Y. Wang, Harnack and functional inequalities for generalized Mehler semigroups, J. Funct. Anal. 203, no. 1, 237-261, 2003.

[14] F.-Y. Wang, Logarithmic Sobolev inequalities on noncompact Riemannian manifolds, Probability Theory Relat. Fields 109, 417-424, 1997.

[15] F.-Y. Wang, Functional Inequalities, Markov Semigroups, and Spectral Theory, Science Press, Beijing 2005.

[16] F.-Y. Wang, Harnack inequality and applications for stochastic generalized porous media equations, Ann. Probab. 35, no. 4, 1333-1350, 2007. 